\title{
Divergências funcionais e estratégias de resistência à seca entre espécies decíduas e sempre verdes tropicais ${ }^{1}$
}

Functional divergences and strategies of resistance to drought between tropical deciduous and evergreen species

\author{
Bruno Cruz de Souza ${ }^{2,6}$, Rafael Silva Oliveira ${ }^{3}$, Francisca Soares de Araújo ${ }^{2}$, André Luiz Alves de Lima ${ }^{4}$ \\ Maria Jesus Nogueira Rodal ${ }^{5}$
}

\begin{abstract}
Resumo
Analisamos a variação funcional entre espécies decíduas (ED) e sempre verdes (ESV) para compreender as divergências nas estratégias de tolerância e evitação à seca. O estudo foi realizado em um fragmento de floresta tropical sazonalmente seca, localizada no munícipio de Pentecoste $\left(3^{\circ} 47^{\prime} \mathrm{S}, 39^{\circ} 16^{\prime} \mathrm{W}\right)$, Ceará, Brasil. Mensuramos 17 traços funcionais foliares em 17 ED e cinco ESV, sendo 12 morfofuncionais, um fenológico e quatro fisiológicos. Verificamos que as ED exibiram maior taxa de fotossíntese por massa $(A \underset{\text { massa }}{)}$, menor longevidade foliar (LF) e massa foliar específica (MFE) quando comparadas às sempre verdes. Esses traços foram considerados traços-chaves preditores das estratégias de evitação e tolerância à seca. As ED e ESV apresentaram uma demanda conflitante entre tolerância à seca e taxa fotossintética, pois a LF foi negativamente correlacionada com à $A_{\text {massa }}$. Embora tenham demonstrado diferenças claras na MFE e LF não observamos diferenças significativas na $A_{\text {área }}$ e $g$, consequentemente, ED e ESV não diferiram na eficiência no uso da água durante o período chuvoso. Apesar da variabilidade substancial dentro do grupo, todas as ED exibem estratégia de evitação à seca enquanto que ESV exibem um conjunto de traços funcionais foliares relacionados a estratégia de tolerância à seca.
\end{abstract}

Palavras-chave: Grupos fenológicos, tolerância à seca, evitação à seca.

\begin{abstract}
We analyzed the functional variation between deciduous and evergreen species to understand the differences across the strategies of avoidance and tolerance to drought. Our study was carried in a fragment of seasonally dry tropical forest located in the city of Pentecoste $\left(3^{\circ} 47^{`} \mathrm{~S}, 39^{\circ} 16^{\prime} \mathrm{W}\right)$, Ceará, Brazil. We measured 17 functional leaf traits in 17 deciduous and five evergreen species, being 12 morphofunctional, one phenological and four physiological. We found that deciduous species exhibited greater $A_{\text {mass }}$, lower leaf lifespan (LL) and leaf mass per area (LMA) than evergreen species. These traits were considered key predictor traits of avoidance and drought tolerance strategies. Deciduous and evergreen species showed a trade-off between drought tolerance and photosynthetic rate, LL was negatively correlated with $A_{\text {mass }}$. Although we have shown clear differences in LMA and LL not observed significant differences in $A_{\text {area }}$ and $g$, consequently, deciduous species and evergreen species did not find significant differences in WUE and WUE. Despite substantial variability within the group, all deciduous species fall predominantly in the drought avoidance strategy, while evergreen species display a suite of traits related to drought tolerance.
\end{abstract}

Key words: Phenological groups, drought tolerance, drought avoidance.

\footnotetext{
${ }^{1}$ Parte da tese de doutorado do primeiro autor.

${ }^{2}$ Universidade Federal do Ceará, Programa de Pós-Graduação em Ecologia e Recursos Naturais, Depto. Biologia, 60455-970, Fortaleza, CE, Brasil.

${ }^{3}$ Universidade Estadual de Campinas, Inst. Biologia, Depto. Biologia Vegetal, 13083-970, Campinas, SP, Brasil.

${ }^{4}$ Universidade Federal Rural de Pernambuco, Unidade acadêmica de Serra Talhada, 56900-000, Serra Talhada, PE, Brasil.

${ }^{5}$ Universidade Federal Rural de Pernambuco, Depto. Biologia, 52171-900, Recife, PE, Brasil.

${ }^{6}$ Autor para correspondência: brunoecologo@gmail.com
} 


\section{Introdução}

Espécies de árvores com diferentes estratégias funcionais, sempre verdes e decíduas, coocorrem em florestas sazonalmente secas (Sobrado 1993; Eamus 1999; Givnish 2002; Fu et al. 2012). Espécies decíduas evitam a seca, produzindo folhas pouco longevas e transpirando a taxas insignificantes na estação seca (Chabot \& Hicks 1982; Eamus 1999). Por outro lado, espécies sempre verdes toleram à seca, apresentando elevada longevidade foliar e fluxo transpiracional durante todo ano (Chabot \& Hicks 1982; Eamus 1999; Mediavilla \& Escudero 2003).

Embora espécies decíduas e sempre verdes demonstrem diferenças entre o uso de água e nutrientes (Kikuzawa 1991; Givnish 2002; Ishida et al. 2010), ainda não é claro se durante o período de maior disponibilidade hídrica essas diferenças são mantidas. Em relação às trocas gasosas e uso da água, espécies decíduas são menos conservativas no uso da água, transpiram a elevadas taxas no período chuvoso em troca de maior ganho de carbono (Mediavilla \& Escudero, 2003). Em contrapartida, espécies sempre verdes são mais conservativas no uso da água, exibem baixa condutância estomática e grande sensibilidade estomática quando sujeitas a alta demanda evaporativa (Mediavilla \& Escudero 2003). Porém, essas divergências entre espécies decíduas e sempre verdes não são demonstradas de forma consensual (ver Damesin et al. 1998; Bucci et al. 2005).

A longevidade foliar é um traço funcional que indica divergências no ganho de carbono e taxa de crescimento entre os grupos de espécies decíduas e sempre verdes (Kikuzawa 1991; Williams-Linera 2000; Ishida et al. 2010). Geralmente esse traço é correlacionado positivamente com a massa foliar e negativamente com a capacidade fotossintética e o conteúdo foliar de nitrogênio e fósforo (Reich et al. 1992; Williams-Linera 2000; Cordell et al. 2001; Wright et al. 2004). Espécies decíduas apresentam menor massa foliar específica e maior taxa fotossintética por unidade de massa se comparadas com espécies sempre verdes (Fu et al. 2012). Espécies sempre verdes apresentam elevada massa foliar, pois, investem grande parte do carbono assimilado na construção das folhas (Wilson et al. 1999). O alto investimento aumenta a resistência à difusão do $\mathrm{CO}_{2} \mathrm{e}$, consequentemente, reduz a capacidade fotossintética em espécies sempre verdes (Ishida et al. 2008). Portanto, duas estratégias ecológicas são identificadas, espécies decíduas que constroem folhas pouco longevas com alta capacidade fotossintética e, espécies sempre verdes que possuem folhas longevas e elevada massa foliar, porém, com baixa capacidade fotossintética (Wright et al. 2006).

Dados empíricos demonstram que o comportamento fenológico das espécies está diretamente associado ao tipo de estratégia em resposta à seca e a forma de utilização da água. Espécies sempre verdes apresentam área foliar reduzida e baixa condutância estomática em resposta ao limite de água disponível (Dudley 1996; Heschel \& Riginos 2005), exibindo maior eficiência no uso de água. Em contraste, espécies decíduas possuem maior área foliar e condutância estomática (Heschel \& Riginos 2005), porém, com menor eficiência no uso de água. A coocorrência de espécies decíduas e sempre verdes fornece evidências de duas diferentes estratégias de utilização da água (Fu et al. 2012) e resistência à seca (Markesteijn \& Poorter 2009).

Embora existam evidências de que traços foliares morfológicos e fisiológicos estão relacionados às estratégias de resposta à seca, ainda não é claro quais traços podem ser considerados bons preditores dessas estratégias. Por exemplo, em espécies ocorrentes no semiárido brasileiro, Silva et al. (2014) consideraram deciduidade foliar como uma importante estratégia adaptativa à seca, utilizando traços funcionais como densidade da madeira (DBM), área foliar específica (AFE) e espessura foliar (EF) como preditores dessa estratégia, esses autores não conseguiram predizer de forma consistente grupos funcionais de plantas.

Outros estudos recentes realizados no semiárido do Brasil demonstraram que associações entre traços do caule e o comportamento fenológicos das espécies foram essenciais para identificar grupos funcionais, além disso, observaram que esses grupos respondem diferentemente a fatores abióticos como temperatura, fotoperíodo e precipitação (Lima \& Rodal 2010; Lima et al. 2012; Oliveira et al. 2015). No entanto, as diferenças entre traços funcionais foliares e as implicações dessas diferenças nas trocas gasosas e relações hídricas das espécies não foram observadas. Estudos que consideraram aspectos físiológicos das espécies, utilizaram como forma de entender as respostas das plantas à seca, relacionando o potencial hídrico com a eficiência quântica da fotossíntese ou a taxa de transpiração com a alocação de solutos na folha 
(Trovão et al. 2007; Silva et al. 2004), porém, não associaram essas respostas ao comportamento fenológico.

Embora alguns autores já tenham observado associações entre a fenologia foliar e estratégias hídricas em espécies tropicais de ambientais sazonalmente secos (Medina \& Francisco 1994; Eamus 1999; Mediavilla \& Escudero 2003), ainda não há consenso sobre essas associações. Por exemplo, Medina \& Francisco (1994) observaram que a espécie decídua Godmania macrocarpa Hemsley é mais tolerante à seca do que a espécie sempre verde Curatella americana L. mesmo exibindo maior taxa de fotossíntese. Por outro lado, Mediavilla \& Escudero (2003) encontraram que espécies decíduas exibem estratégia menos conservativa no uso da água e maiores taxas de fotossíntese do que espécies sempre verdes que são mais tolerantes aos efeitos negativos da seca.

Nesse contexto, nossos objetivos foram: (1) investigar se espécies decíduas e sempre verdes apresentam variações entre traços funcionais foliares (massa foliar específica, longevidade foliar, taxa de fotossíntese, condutância estomática e eficiência no uso da água) relacionados às estratégias de resistência à seca (evitação e tolerância sensu Levitt 1972) e utilização da água e; (2) identificar traços preditores de estratégias de tolerância e evitação à seca que se relacionam com o comportamento fisiológico das espécies. Nossas hipóteses foram que (1) As espécies decíduas se diferenciam das espécies sempre verdes em um conjunto de traços funcionais foliares que são relacionados com o tipo de estratégia de uso de recursos e; (2) $\mathrm{Na}$ ausência de restrição hídrica espécies decíduas se diferenciam das sempre verdes, exibindo elevada taxa fotossintética e condutância estomática, resultando em menor eficiência no uso da água.

\section{Materiais e Métodos}

Área de estudo

Nossa pesquisa foi desenvolvida na Fazenda Experimental Vale do Curu ( $\left.3^{\circ} 47^{\prime} \mathrm{S}, 39^{\circ} 16^{\prime} \mathrm{W}\right)$, pertencente à Universidade Federal do Ceará (UFC), localizada no município de Pentecoste, Ceará, Brasil (Fig. 1). Os solos são rasos e com pouca capacidade de retenção hídrica. A topografia da região é plana com altitude média de $75 \mathrm{~m}$. O clima da região no sistema de classificação de Köppen-Geiger é BSh - semiárido, seco, com chuvas no verão e seca no inverno (Peel et al. 2007).
A precipitação média anual na última década (2000-2010) foi de $742 \mathrm{~mm}$ e a temperatura média anual em torno de $28,5^{\circ} \mathrm{C}$ (dados da estação meteorológica da fazenda; Fig. 2). O período chuvoso historicamente é concentrado entre três e quatros meses (janeiro e abril), seguido por longo período seco (maio e dezembro). Em 2012, ano que esse estudo foi desenvolvido, a precipitação total foi de $416,9 \mathrm{~mm}$ e a temperatura média de $28,8^{\circ} \mathrm{C}$ (máx. $35^{\circ} \mathrm{C}$ e mín. $22.6^{\circ} \mathrm{C}$ ).

\section{Espécies analisadas}

Demarcamos uma área de $1 / 2$ hectare $\mathrm{e}$ subdividimos em parcelas contíguas de $10 \times 10 \mathrm{~m}$ para amostragem da composição de espécies. Amostra de partes reprodutivas e vegetativas foram coletas e herborizadas, em seguida, o material botânico foi comparado com exsicatas do herbário Prisco Bezerra (EAC) para determinação das espécies. Sistematicamente, selecionamos espécies de árvores e arbustos (diâmetro ao nível do solo > $3 \mathrm{~cm}$ ), sendo 17 decíduas e cinco sempre verdes, totalizando 22 espécies (Tab. 1). De acordo com Oliveira et al. (2015) a duração das folhas nas espécies decíduas varia de 5-6 meses e nas espécies sempre verdes é superior a 11 meses.

\section{Traços funcionais foliares}

No período de dezembro de 2011 a novembro de 2012, em intervalos de 15 dias, observamos o comportamento fenológico das espécies para determinamos a longevidade foliar (LF; dias). A longevidade foliar foi estimada pela média de dias entre as fenofases de brotação e queda foliar. Mensuramos os demais traços foliares de março a abril de 2012, meses que compreendem o período chuvoso na região. De acordo com as observações do comportamento fenológico foliar das espécies, todas as folhas utilizadas nesse estudo foram desenvolvidas nas espécies decíduas no início do período chuvoso (fevereiro) e nas espécies sempre verdes no final da estação seca (janeiro). Cinco indivíduos por espécie com alturas semelhantes foram selecionados. Em cada indivíduo 10 folhas expostas ao sol foram coletadas, colocadas em papéis toalha umedecidos e acondicionadas por duas horas em caixa térmica refrigerada por gelo (Cornelissen et al. 2003). Conforme o protocolo proposto por Cornelissen et al. (2003), as folhas foram reidratadas em câmara úmida por 6 à 8 horas, logo após, determinamos a massa fresca (MF; $\mathrm{mg})$. A área foliar $\left(\mathrm{AF} ; \mathrm{cm}^{2}\right)$ foi determinada 



Figura 1 - Mapa do Brasil com a localização do estado do Ceará na região nordeste (a. C 2009 Google, Map Data C 2009 Europa Technologies) e localização do território da cidade de Pentecoste (b. (c) 2009 Google, Map Data (c) 2009 Europa Technologies). Fotografias da área de estudo na (1/2 hectare) fazenda experimental Vale do Curú no período chuvoso (c) e seco (d).

Figure 1 - Map of the Brazil with the Ceará state localization on the Northeast region (a. (C) 2009 Google, Map Data ( 2009 Europa Technologies) and territorial location of the Pentecoste city (b. (C) 2009 Google, Map Data (C) 2009 Europa Technologies). Photographs of the study area in (1/2 hectare) experimental farm Vale do Curú in wet (c) and dry seasons (d).

através da digitalização das folhas em scanner de mesa e as imagens analisadas no software image $J$ v.1.44. Para estimar a espessura foliar (EF; em $\mathrm{mm}$ ) e tamanho do pecíolo (TP; em $\mathrm{cm}$ ) utilizamos paquímetros digitais, em seguida, as folhas foram colocadas para secagem em estufa de ar circular por 48 horas em temperatura de $70^{\circ} \mathrm{C}$ até atingirem a massa seca constante (Poorter 2009).

Com bases nessas medidas calculamos: área foliar específica (AFE; área foliar fresca/massa seca; em $\mathrm{mm}^{2} \mathrm{mg}^{-1}$ ), massa foliar específica (MFE; 1/AFE; em $\mathrm{mg} \mathrm{mm}^{-2}$ ), conteúdo saturado de água (CSA; massa fresca saturada - massa seca/ massa seca; em $\mathrm{g} \mathrm{g}^{1}$ ) (Hao et al. 2010), massa seca foliar (MSF; massa fresca saturada/massa seca; em mg $\mathrm{g}^{-1}$ ), suculência foliar (SF; massa fresca saturada massa seca/área foliar; em $\mathrm{g} \mathrm{m}^{-2}$ ) (Mantovani 1999) e densidade foliar (DF; massa seca/(área foliar * espessura foliar); em $\mathrm{mg} \mathrm{mm}^{-3}$ ) (Poorter 2009).

$\mathrm{O}$ conteúdo de clorofila foi estimado utilizando medidor de clorofila SPAD (Minolta SPAD 502 D Spectrum Technologies Inc., Plainfield, IL, USA). Determinamos a concentração de clorofila por área $\left(\mathrm{C}_{\text {area}}\right.$; Conteúdo de clorofila lárea foliar; em $\mu \mathrm{mol} \mathrm{m}{ }^{2}$; Poorter 2009). Para o conteúdo de clorofila utilizamos o modelo polinomial para árvores tropicais: Conteúdo Clorofila $=0.664 * \mathrm{SPADi}+0.012 * \mathrm{SPADi}^{2} ;$ Coste et al. 2010). Para testarmos a adequação da equação adotada, comparamos o conteúdo de clorofila 




Figura 2 - Diagrama climático Walter e Lieth (1960) para a fazenda experimental Vale do Curu, Pentecoste, Ceará, Brasil. Dados climáticos (temperatura máxima, mínima e precipitação) da última década (2000-2010). Figure 2 - Climatic diagram Walter and Lieth (1960) for Experimental Farm Vale of Curu, Pentecoste, Ceará, Brazil. Climatic data (maximum and minimum temperature and precipitation) of the last decade (2000-2012).

determinado pela equação utilizada por Poorter (2009). Não encontramos diferenças significativas entre o conteúdo de clorofila determinado pelas duas equações $(\mathrm{P}=0.45)$. Utilizando as mesmas folhas amostradas para mensuração dos traços morfológicos, determinamos por indivíduo o conteúdo de nitrogênio por massa seca $\left(\mathrm{N}_{\text {massa }}\right.$; $\%$ massa seca) e fósforo por massa seca ( $\mathrm{P}_{\text {massasa }}$; $\%$ massa seca). A concentração de nitrogênio foliar total foi determinada através da utilização do método micro-Kjeldahl (Bremmer 1965). Para a determinação do conteúdo total de fósforo utilizamos após destilação uma mistura triácida de ácidos nítrico, sulfúrico e perclórico, na proporção de 10:1:2. O fósforo total no extrato foi estabelecido por colorimetria, a $410 \mathrm{~nm}$, utilizando vanadomolibdato de amônia (Allen 1989).

\section{Comportamento fisiológico}

No período chuvoso (março e abril 2012) em cinco folhas expandidas, expostas ao sol e sem danos de cinco individuo por espécie mensuramos a taxa de fotossíntese por área $\left(A_{\text {área }} ; \mu \mathrm{mol} \mathrm{CO} \mathrm{m}^{-2}\right.$ $\left.\mathrm{s}^{-1}\right)$ e a condutância estomática $\left(g_{s} ; \mathrm{mol} \mathrm{m}^{-2} \mathrm{~s}^{1}\right)$ entre
08:00 e 12:00 h. Utilizamos um medidor portátil de fotossíntese (Modelo LI-6400XT; LI-COR Inc., Lincoln, NE, USA). Na câmara do medidor portátil mantivemos as folhas por aproximadamente $120 \mathrm{~s}$, a concentração de $\mathrm{CO}_{2}$ foi fixada em 400ppm e a taxa de fluxo de $400 \mu \mathrm{mol} \mathrm{s}^{-1}$. A umidade relativa do ar em condições naturais variou entre $50 \%$ a $70 \%$ e a temperatura entre 25 e $32^{\circ} \mathrm{C}$. Após medidas da radiação fotossinteticamente ativa em condições de pleno sol, estabelecemos um valor fixo de 1800 $\mu \mathrm{mol}$ fótons $\mathrm{m}^{-2} \mathrm{~s}^{-1}$ dentro da câmara com uso de luz artificial. Determinamos ainda a eficiência intrínseca no uso da água (EUA; $A_{\text {area }} / g_{s} ; \mu \mathrm{mol} \mathrm{CO}$ $\mathrm{mol} \mathrm{H}_{2} \mathrm{O}^{-1}$ ), a eficiência instantânea no uso da água $\left(\mathrm{EUA}_{\mathrm{i}} ; A_{\text {area }} / E \mu \mathrm{mol} \mathrm{CO} \mathrm{mmol} \mathrm{H}_{2} \mathrm{O}^{-1} ; E\right.$ - taxa de transpiração) e a taxa de fotossíntese por unidade de massa $\left(A_{\text {massa }} ; A_{\text {massa }}=A_{\text {arrea }} / \mathrm{MFE} ; \mathrm{em} \mathrm{nmol} \mathrm{g} \mathrm{s}^{-1}\right)$.

\section{Análises estatísticas}

Como forma de comparar as diferenças em traços funcionais foliares entre espécies decíduas e sempre verdes, adotamos o teste $t$ para amostras independentes. Em caso de hipótese nula rejeitada para normalidade (Shapiro Wilk's) e igualdade de variância (teste de Bartlett) transformamos o valor do traço para $\log _{10}$ antes de aplicarmos o teste. Realizamos análises de componentes principais (ACP) com auxílio do software PC-ORD 6 (Multivariate Analysis of Ecological Data, Oregon, U.S.A) para identificarmos possíveis agrupamentos ou gradientes de variação funcional entre as espécies. Utilizamos modelos de regressão para verificar as correlações e variações na amplitude da taxa de fotossíntese, longevidade foliar, área foliar específica e condutância estomática. A eficiência intrínseca e instantânea no uso da água foi comparada entre os grupos de espécies decíduas e sempre verdes por análise de variância (ANOVAone way) seguida de teste post-hoc de Tukey $(\alpha=0,05)$. Todas as análises estatísticas foram realizadas no ambiente R v.2.15.1 (R Development Core Team, 2012) e no software PAST (Hammer et al. 2001).

\section{Resultados}

Diferenças funcionais entre espécies decíduas e sempre verdes

Nós observamos que espécies decíduas e sempre verdes diferiram em traços funcionais foliares relacionados às estratégias de evitação e tolerância à seca. Espécies decíduas apresentaram $\mathrm{AF}, \mathrm{AFE}, \mathrm{CSA}$ e $A_{\text {massa }}$ significativamente maiores 
Tabela 1 - Grupos fenológicos e formas de crescimento das espécies selecionadas para amostragem de traços funcionais foliares.

Table 1 - Phenology groups and growth form of the species selected for sampling of the leaf functional traits.

\begin{tabular}{lcc}
\hline Espécies & Grupo Fenológico & Forma de Crescimento \\
\hline Aspidosperma pyrifolium Mart. & Decídua & Árvore \\
Cordia oncocalyx Allemão & Decídua & Árvore \\
Cordia trichotoma (Vell.) Arrabida ex Steudel. & Decídua & Árvore \\
Cynophalla flexuosa L. & Sempre Verde & Arbusto \\
Manihot carthaginensis (Jacq.) Müll. Arg. & Decídua & Arbusto \\
Cochlospermum vitifolium (Willd.) Spreng. & Decídua & Árvore \\
Amburana cearensis (Allemão) A.C. Sm. & Decídua & Árvore \\
Commiphora leptophloeos (Mart.) J.B. Gillett & Decídua & Árvore \\
Combretum leprosum Mart. & Decídua & Arbusto trepador \\
Croton blanchetianus Baill. & Decídua & Árvore \\
Sebastiania macrocarpa Müll. Arg. & Decídua & Árvore \\
Anadenanthera colubrina (Vell.) Brenan & Decídua & Árvore \\
Bauhinia cheilantha (Bong.) D. Dietr. & Decídua & Árvore \\
Libidibia ferrea (Mart.) L.P.Queiroz & Sempre Verde & Árvore \\
Mimosa caesalpiniifolia Benth. & Decídua & Árvore \\
Piptadenia stipulacea (Benth.) Ducke & Decídua & Árvore \\
Piptadenia viridiflora (Kunth) Benth. & Sempre Verde & Árvore \\
Poincianella bracteosa (Tul.) L.P.Queiroz & Decídua & Árvore \\
Lafoensia pacari A.St.-Hil & Decídua & Árvore \\
Helicteres heptandra L.B.Sm. & Decídua & Arbusto \\
Zizyphus joazeiro Mart. & Sempre Verde & Árvore \\
Ximenia americana L. & Sempre Verde & Árvore \\
\hline
\end{tabular}

do que espécies sempre verdes (Tab. 2). O grupo de espécies sempre verdes demonstrou LF, MFE, $\mathrm{DF}, \mathrm{C}_{\text {area }}$ e CMSF significativamente maiores do que o grupo das espécies decíduas (Tab. 2). Nos outros traços foliares mensurados (TP, EF, SF, $A_{\text {area, }}$ $g_{s}$, EUA, $\mathrm{N}_{\text {massa }}$ e $\mathrm{P}_{\text {massa }}$ ) não observamos diferenças significativas entre os grupos (Tab. 2).

\section{Análises de componentes principais}

Nós encontramos evidências de um conjunto específico de traços funcionais foliares que categoriza as espécies em grupos segundo a fenologia foliar e estratégia de resistência à seca. $\mathrm{Na}$ análise de componentes principais (ACP), o primeiro e segundo componente representados no eixo 1 e 2 explicam $37,8 \%$ e $17,2 \%$ da variação de 17 traços foliares (o terceiro eixo apenas 13\%). Os traços que constituíram o eixo 1 estão relacionados com a longevidade, investimento de carbono na construção das folhas e capacidade fotossintética (Fig. 3a). Os valores dos traços LF, MFE, DF e CMSF indicam associações com os scores do lado positivo do eixo 1 (Fig. 3a). No lado negativo, os scores foram associados apenas com os valores dos traços $A_{\text {massa }}$ e AFE (Fig. 3a). No segundo eixo, os traços $(\mathrm{SF}, \mathrm{CSA}$ e $\mathrm{EF})$ relacionados à capacidade hídrica das folhas foram associados apenas aos scores negativos do eixo (Fig. 3a).

A posição das espécies plotadas na ACP reflete uma clara distinção entre duas estratégias de resistência à seca baseada apenas em traços foliares (Fig. 3b). O grupo formado por cinco espécies sempre verdes (valores positivos do eixo 1) exibiram elevada longevidade e massa foliar, mas, ao longo do eixo 2 se diferenciam em relação a capacidade hídrica das folhas (Fig. 3b). As espécies decíduas representam um gradiente de variação funcional, no entanto, 12 espécies exibiram traços foliares que refletem alta capacidade fotossintética (valores negativos do eixo 1) e assim, como nas espécies sempre verdes diferenciam-se apenas em relação a capacidade hídrica (Fig. 3b).

\section{Ecofisiologia e estratégias à seca}

Nós encontramos que espécies decíduas e sempre verdes exibem claramente uma demanda conflitante entre duração das folhas e taxa fotossintética. A taxa de fotossíntese por massa 
foliar $\left(A_{\text {massa }}\right)$ declina com o aumento na longevidade foliar (LF) sendo significativamente maior nas espécies decíduas do que nas espécies sempre verdes $\left(\mathrm{R}^{2}=0,51 P<0,001\right.$; Fig. 3a). Nas espécies decíduas, a LF e $A_{\text {massa }}$ apresentou elevada variação, diferentemente das espécies sempre verdes que demonstraram pouca variação (Fig. 4a). A $A_{\text {massa }}$ foi correlacionada com a condutância estomática $\left(g_{s}\right)\left(\mathrm{R}^{2}=0,43 P<0,001 ;\right.$ Fig. 4b) e área foliar (AF) $\left(\mathrm{R}^{2}=0,70 P<0,001\right.$; Fig. $\left.4 \mathrm{c}\right)$. As espécies decíduas exibiram maior $g_{s}$ do que as espécies sempre verdes, porém, essa diferença entre os grupos não foi significativa estatisticamente (Fig. 4b).

Nós encontramos que durante o período chuvoso (sem restrições hídricas) espécies decíduas e sempre verdes não se diferenciam em relação ao uso da água. A eficiência instantânea no uso da água $\left(\mathrm{EUA}_{\mathrm{i}}\right)$ e a eficiência intrínseca no uso da água (EUA) não foi significativamente diferente entre espécies decíduas e sempre verdes (Fig.5; $F=0,2611 P=0,61 ; F=0,04 P=0,84)$.

\section{Discussão}

Neste estudo, nós encontramos que espécies decíduas e sempre verdes se diferenciam nos traços funcionais foliares que refletem claramente a ocorrência de duas estratégias de resistência à seca. Além disso, nós fornecemos informações sobre o comportamento fisiológico das espécies quando a disponibilidade hídrica não restringe as trocas gasosas e uso da água. As espécies decíduas apresentaram maior taxa fotossintética por unidade de massa, refletindo baixo investimento de carbono na construção da folha, porém, com alta capacidade na assimilação de carbono. Por outro lado, espécies sempre verdes exibiram elevada longevidade foliar

Tabela 2 - Comparação de traços foliares entre 17 espécies decíduas e cinco sempre verdes (médias \pm erro padrão). Table 2 - Comparison of the leaf traits between 17 species deciduous and five evergreens (mean \pm standard error).

\begin{tabular}{|c|c|c|c|c|c|}
\hline Traços foliares & Unidades & Decíduas & Sempre Verdes & $t$ & $P$ \\
\hline LF & dias & $159,0 \pm 10,7$ & $326,8 \pm 17,4$ & $-7,61$ & 0,001 \\
\hline $\mathrm{AF}$ & $\mathrm{cm}^{2}$ & $85,0 \pm 16,6$ & $33,4 \pm 13,0$ & 2,12 & 0,046 \\
\hline AFE & $\mathrm{mm} \mathrm{mg}^{-2}$ & $28,3 \pm 2,31$ & $11,3 \pm 1,3$ & 5,93 & 0,001 \\
\hline TP & $\mathrm{cm}$ & $27,8 \pm 9,5$ & $10,7 \pm 6,0$ & 1,03 & 0,313 \\
\hline MFE & $\mathrm{mg}^{-1} \mathrm{~mm}^{2}$ & $0,03 \pm 0,0$ & $0,09 \pm 0,0$ & $-6,79$ & 0,001 \\
\hline $\mathrm{EF}$ & $\mathrm{mm}$ & $0,21 \pm 0,02$ & $0,21 \pm 0,05$ & $-0,02$ & 0,981 \\
\hline $\mathrm{DF}$ & $\mathrm{g} \mathrm{cm}^{-3}$ & $0,23 \pm 0,03$ & $0,53 \pm 0,09$ & $-3,95$ & 0,001 \\
\hline MSF & $\mathrm{mg} \mathrm{g}^{-1}$ & $315,2 \pm 19,7$ & $463,3 \pm 28,9$ & $-3,71$ & 0,001 \\
\hline CSA & $\mathrm{g} \mathrm{g}^{1}$ & $2,48 \pm 0,2$ & $1,22 \pm 0,15$ & 3,17 & 0,004 \\
\hline SF & $\mathrm{g} \mathrm{m}^{-2}$ & $90,8 \pm 32,4$ & $114,9 \pm 24,3$ & $-1,24$ & 0,225 \\
\hline $\mathrm{C}_{\text {area }}$ & $\mu \mathrm{mol} \mathrm{m}{ }^{2}$ & $32,0 \pm 1,5$ & $43,3 \pm 6,4$ & $-2,61$ & 0,016 \\
\hline$A_{\text {arrea }}^{\text {area }}$ & $\mu \mathrm{mol} \mathrm{CO} \mathrm{m}^{-2} \mathrm{~s}^{-1}$ & $8,29 \pm 0,6$ & $7,38 \pm 0,9$ & 0,65 & 0,518 \\
\hline$A_{\text {massa }}$ & $\mathrm{nmol} \mathrm{CO}_{2} \mathrm{~g}^{-1 \mathrm{~s}-1}$ & $232,2 \pm 25,6$ & $80,8 \pm 10,9$ & 3,12 & 0,005 \\
\hline $\mathrm{g} s$ & $\mathrm{~mol} \mathrm{H}_{2} \mathrm{O} \mathrm{m}^{-2} \mathrm{~s}-1$ & $0,37 \pm 0,02$ & $0,31 \pm 0,03$ & 1,18 & 0,255 \\
\hline EUA & $\mu \mathrm{mol} \mathrm{CO}{ }_{2} \mathrm{~mol}^{-1} \mathrm{H}_{2} \mathrm{O}$ & $23,0 \pm 1,7$ & $24,8 \pm 3,8$ & $-0,46$ & 0,645 \\
\hline $\mathrm{N}_{\text {massa }}$ & $\mathrm{mg} \mathrm{g}^{-1}(\%)$ & $2,48 \pm 0,1$ & $2,19 \pm 0,1$ & 1,17 & 0,254 \\
\hline $\mathrm{F}_{\text {massa }}$ & $\operatorname{mg~g}^{-1}(\%)$ & $0,20 \pm 0,02$ & $0,17 \pm 0,02$ & 0,49 & 0,629 \\
\hline
\end{tabular}

Média de dois grupos fenológicos comparados por teste $t$ para amostras independentes. Valores de $P<0,05$ exibidos em negrito. LF, longevidade foliar; AF, área foliar; AFE, Área foliar específica; TP, tamanho do pecíolo; MFE, massa foliar específica; EF, espessura foliar; DF, densidade foliar; MSF, massa seca foliar; CSA, conteúdo saturado de água; $\mathrm{SF}$, suculência foliar; $\mathrm{C}_{\text {área }}$, conteúdo de clorofila por área; $\mathrm{A}_{\text {área }}$, taxa de fotossíntese por área; $\mathrm{A}_{\text {massa }}$, taxa de fotossíntese por unidade de massa; $\mathrm{g}_{\mathrm{s}}$, condutância estomática; EUA, eficiência intrínseca no uso da água; $\mathrm{N}_{\text {massa }}$, nitrogênio por unidade de massa; $\mathrm{F}$, fósforo por unidade de massa.

Means the two phenological groups compared in test-t for independent samples. Values of $\mathrm{P}<0.05$ showing in bold. LF, leaf lifespan; AF, leaf area; AFE, specific leaf area; TP, petiole length; MFE, leaf dry mass per area; EF, leaf thickness; DF, leaf density; MSF, leaf dry matter; CSA, leaf saturated water content; $\mathrm{SF}$, leaf succulence; $\mathrm{C}_{\text {área }}$, chlorophyll per unit leaf area; $\mathrm{A}_{\text {área }}$ leaf maximum area-based photosynthesis rate; A , leaf maximum mass-based photosynthesis rate; gs, maximum stomatal conductance; EUA, water-use intrinsic efficiency; $\mathrm{N}_{\text {massa }}$, nitrogen per unit mass; $\mathrm{F}_{\text {massa }}$, phosphorus per unit mass. 
o que representa alto investimento de carbono na construção das folhas. Esses resultados são consistentes aos encontrados em outros estudos que igualmente compararam traços funcionais entre espécies decíduas e sempre verdes em regiões semiáridas (Ackerly 2004; Franco et al. 2005; Fu et al. 2012). As diferenças em traços foliares específicos como LF, AFE, MFE, $A_{\text {massa }} \mathrm{e}$ CSA demonstraram que esses grupos fenológicos se diferenciam em mecanismos fisiológicos como trocas gasosas e uso da água, portanto, apresentam estratégias distintas de resistência à seca (ver Ackerly 2004).

Embora não observamos diferença na $A_{\text {area }}$ e $g_{s}$ entre espécies decíduas e sempre verdes, espécies decíduas exibiram baixa MFE o que

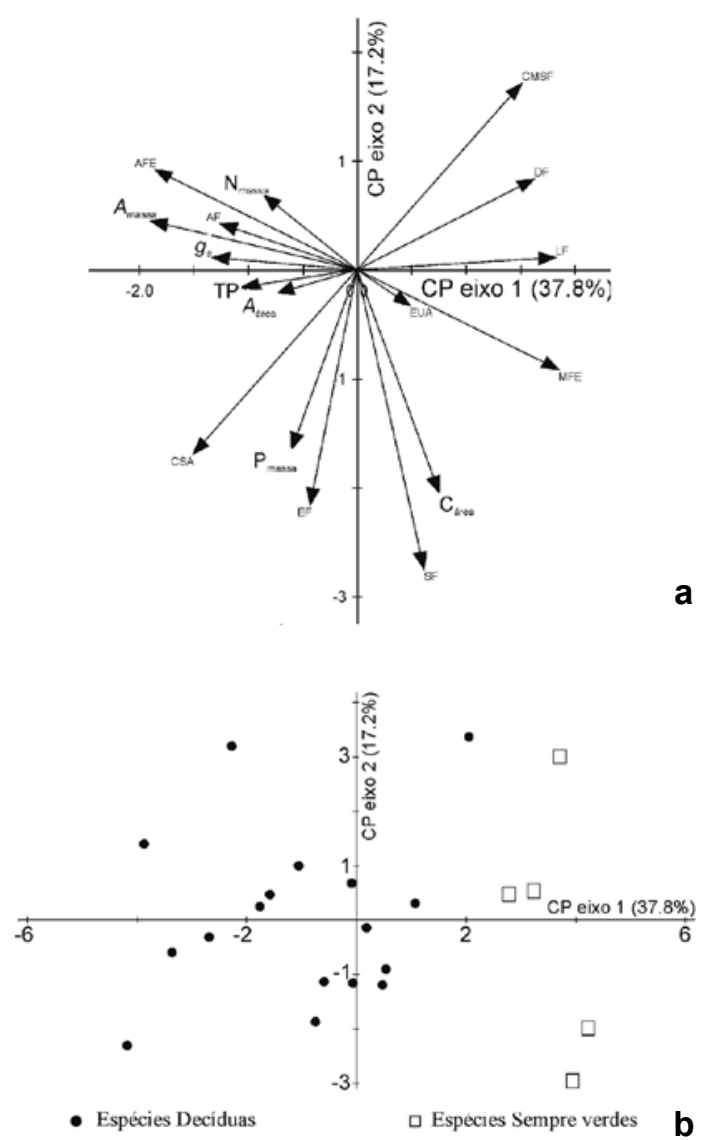

Figura 3 - Ordenação de 17 traços funcionais foliares ao longo de dois eixos de componentes principais (a). Distribuição das espécies decíduas e sempre verdes no espaço ao longo do eixo 1e 2 (b).

Figure 3 - Ordination of the 17 leaf functional traits along two principal component axes (a). Distribution of the species deciduous and evergreen in space along axis 1 axis 2 (b). resulta em elevada $A_{\text {massa }}$ em comparação com as espécies sempre verdes. Outros estudos também demonstraram que diferenças na capacidade fotossintética desses grupos são mais evidentes quando utilizamos $A_{\text {massa }}$ ao invés da $A_{\text {area }}$ (Ackerly 2004; Fu et al. 2012), sendo essa variação entre a taxa de assimilação por área e massa uma importante regra na limitação de água em ambientes semiáridos e áridos (Ackerly 2004).

No período chuvoso as folhas de espécies decíduas demonstraram elevado conteúdo de água (maior CSA) o que sugere uma maior capacidade nas trocas gasosas do que as espécies sempre verdes. Além disso, a maior taxa de fotossíntese em espécies decíduas durante período de alta disponibilidade hídrica, indica a existência da relação de custo benefício na construção das folhas. Essa relação sugere que espécies decíduas mantem uma alta concentração de nitrogênio foliar para manter alta taxa de assimilação de $\mathrm{CO}_{2}$ que é fixado em grande quantidade durante o curto tempo de vida da folha (Eamus 1999). No período seco, essas espécies perdem as folhas para evitar a perda excessiva de água (Borchert 1994; Lima \& Rodal, 2010; Lima et al. 2012). Em contrapartida, espécies sempre verdes mantém as folhas e as trocas gasosas mesmo sob baixos potenciais hídricos, exibindo clara tolerância ao déficit hídrico (Odening et al. 1974).

Neste estudo demonstramos evidências que espécies decíduas e sempre verdes exibem uma demanda conflitante entre tolerância à seca e taxa fotossintética da folha (Fig. 4a). A LF, traço que demonstra tolerância à seca, foi negativamente correlacionado com a $A_{\text {massa, }}$ que reflete a capacidade fotossintética da folha. Espécies decíduas exibem alta capacidade fotossintética e duração curta das folhas. Por outro lado, espécies sempre verdes constroem folhas com alto investimento de carbono que possibilita maior LF, porém, reduz sua capacidade fotossintética (Eamus 1999; Ishida et al. 2006).

Além disso, diferenças pronunciadas na $g_{s}$ entre espécies decíduas e sempre verdes são comumente descritas em diversos ecossistemas sazonalmente secos (Chen et al. 2009; Mediavilla \& Escudero, 2003; Ishida et al. 2006) e são interpretadas com uma demanda conflitante entre LF e eficiência hidráulica (Sobrado 1993; Ishida et al. 2010; Fu et al. 2012). Embora não observamos diferenças significativas na $g_{s}$ entre espécies decíduas e sempre verdes, acreditamos que 

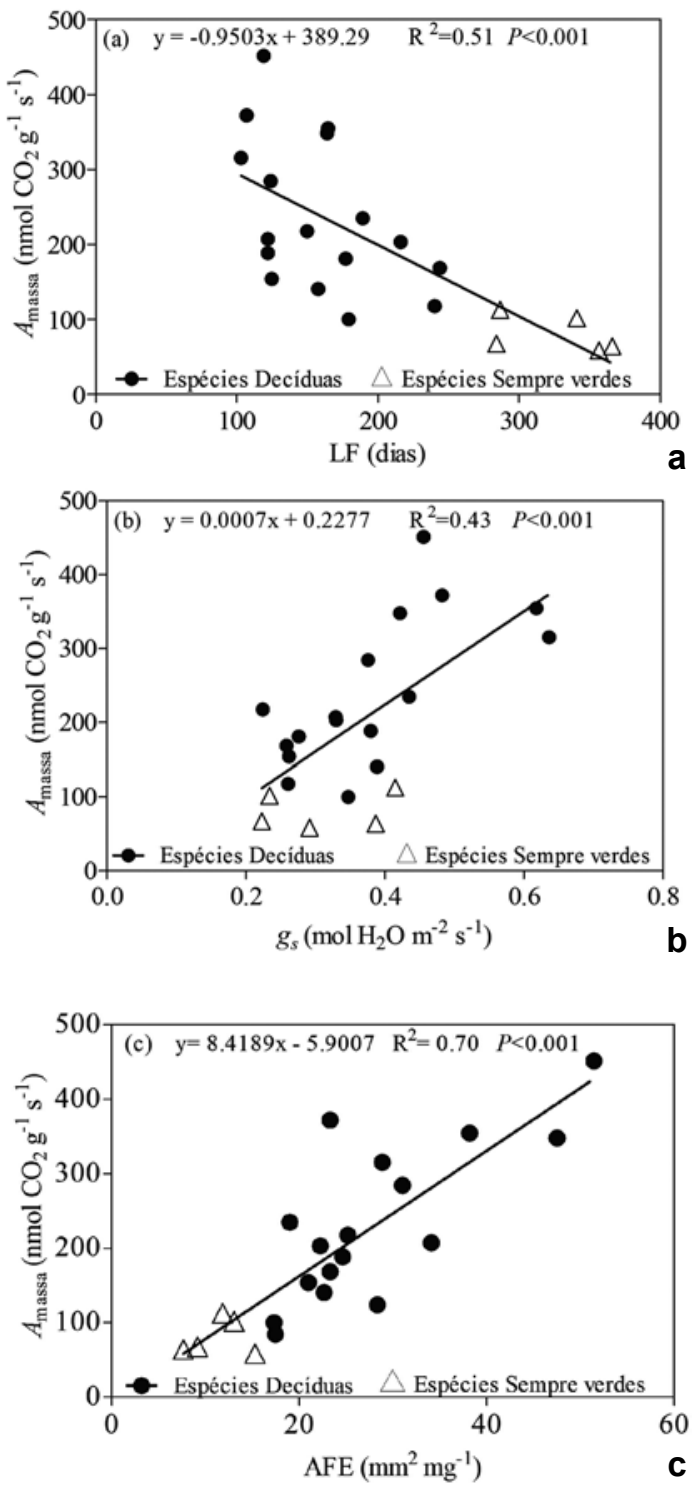

Figura 4 - Regressão linear entre taxa de fotossíntese por unidade de massa $(A)$ massa $)$ e longevidade foliar (LF) em (a), condutância estomática $(g s)$ em (b) e área foliar específica (AFE) em (c). Nota: Média de cinco indivíduos por espécie; Círculos pretos - espécies decíduas; triângulos abertos - espécies sempre verdes. Figure 4 - Linear regression between assimilation rate based mass (Amass) and leaf lifespan (LLS) in (a), stomatal conductance $(\mathrm{gs})$ in (b) and specific leaf area (SLA). Note: Mean of five individuals for specie; Black circles-deciduous species; open triangles - evergreen species.

a maior taxa fotossintética por unidade de massa nas espécies decíduas, ocorreu possivelmente por que essas espécies exibiram menor massa foliar por unidade de área, o que representa uma barreira física a difusão do $\mathrm{CO}_{2}$. Folhas com alto MFE apresentam grande espessura foliar, espessamento das paredes das células do mesófilo e alta densidade de células no mesófilo, essas características resultam em menor condutância de $\mathrm{CO}_{2}$ no interior da folha com redução significativa na taxa de fotossíntese (Kogami et al. 2001).

Espécies sempre verdes tolerantes à seca são mais conservativas no uso da água e exibem, maior sensibilidade estomática aos efeitos da seca do que espécies decíduas que evitam à seca (Mediavilla \& Escudero 2003). No nosso estudo, não observamos diferenças na eficiência do uso da água entre espécies decíduas e sempre verdes (Fig. 5). Isso sugere que as diferenças entre as estratégias de evitação e tolerância à seca são primariamente determinadas por diferenças na capacidade hidráulica, traços relacionados à fotossíntese e tolerância das folhas ao estresse hídrico (Fu et al. 2012). Corroborando como a nossa primeira hipótese, as diferenças entre traços foliares chaves como LF, MFE e $A_{\text {massa }}$ fornecem evidências que esses grupos exibem duas estratégias distintas. As espécies decíduas exibiram curta LF, baixa MFE e elevada $A_{\text {massa }}$ (estratégia de evitação), enquanto que espécies sempre verdes demonstraram alta LF e MFE, porém, ao custo de baixa $A_{\text {massa }}$ (estratégia de tolerância).

Durante o período de disponibilidade hídrica, diferenças em relação a EUA, EUA $\mathrm{e}_{\mathrm{i}} g_{s}$ entre espécies decíduas e sempre verdes não foram observadas, refutando nossa segunda hipótese. Acreditamos que outros mecanismos e traços funcionais não detectados nesse estudo estão relacionados com o uso da água e comportamento estomático dessas espécies. Alguns trabalhos demonstraram que diferenças na EUA e $g_{s}$ entre esses grupos está diretamente relacionada com as respostas estomáticas aos efeitos negativos da seca para manutenção do estado hídrico da planta (Mediavilla \& Escudero, 2003; Bucci et al. 2004; Bucci et al. 2005) ou associada a órgãos que exibem elevada capacidade de armazenamento de água (Bucci et al. 2004; Zane et al. 2010). Desta forma, sugerimos que estudos posteriores investiguem as diferentes associações de traços funcionais com a fenologia foliar e estado hídrico em espécies decíduas e sempre verdes, identificando essencialmente como diferem essas estratégias de resistência a seca (evitação e tolerância) em escala estacional. 


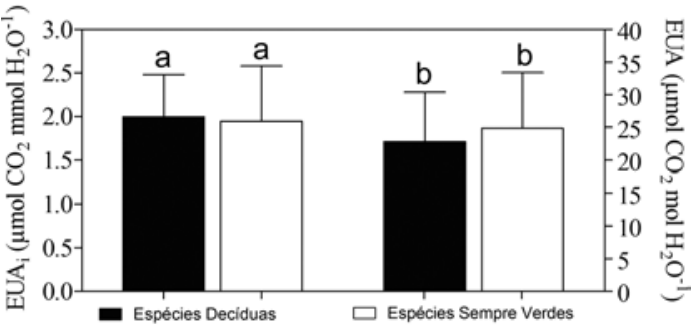

Figura 5 - Comparação entre eficiência intrínseca no uso da água (EUA) e eficiência instantânea no uso da água (EUA) em espécies decíduas e sempre verdes. Nota: Barras pretas - espécies decíduas; barras brancas-espécies sempre verdes; Letra iguais indicam que não diferenças entre os grupos (Teste de Tukey 5\% de significância).

Figure 5 - Comparison between water use efficiency intrinsic (WUE) and water use efficiency instantaneous (WUEi) in deciduous and evergreen species. Note: Black bars-deciduous species; white bars - evergreen species; Equal letters indicate no difference between groups (Tukey test 5\% significance).

\section{Agradecimentos}

À Coordenação de Aperfeiçoamento de Pessoal de Nível Superior (Capes), a concessão da bolsa de estudos do doutorando B.C. Souza. À CAPES, através dos Programas Nacionais de Cooperação Acadêmica (PROCAD 157/2007), e o Conselho Nacional de Desenvolvimento Científico e Tecnológico (Casadinho/Procad, processo $\left.\mathrm{n}^{\circ} 552213 / 2011-0\right)$, o suporte financeiro para realização do estágio de Doutorado Sanduíche de B.C. Souza na Universidade Estadual de Campinas (UNICAMP/São Paulo). A E.C.D Carvalho e a C.C. Oliveira, a ajuda nos trabalhos de campo. Aos coordenadores da Fazenda Experimental Vale do Curu, pertencente a Universidade Federal do Ceará, por permitirem desenvolver os trabalhos de campo e o apoio logístico.

\section{Referências}

Ackerly, D. 2004. Functional strategies of chaparral shrubs in relation to seasonal water deficit and disturbance. Ecological Monographs 74: 25-44.

Allen, S.E. 1989. Chemical analysis of ecological materials. Blackwell Scientific Publications, Oxford. 565p.

Borchert, R. 1994. Soil and stem water storage determine phenology and distribution of tropical dry forest trees. Ecology 75: 1437-1449.

Bremner, J.M. 1965. Total Nitrogen. In: Page, A.L. Methods of soil analysis. Part 2. Chemical and microbiological properties. American Society of Agronomy, Soil Science Society of America, Madison, Wisconsin. Pp. 1149-1178.

Bucci, S.; Goldstein, G.; Meinzer, F.; Scholz, F.; Franco, A. \& Bustamante, M. 2004. Functional convergence in hydraulic architecture and water relations of tropical savanna trees: from leaf to whole plant. Tree Physiology 24: 891-899.

Bucci, S.; Goldstein, G.; Meinzer, F.; Franco, A.; Campanello, P. \& Scholz, F. 2005. Mechanisms contributing to seasonal homeostasis of minimum leaf water potential and predawn disequilibrium between soil and plant water potential in Neotropical savanna trees. Trees 19: 296-304.

Chabot, B. \& Hicks, D. 1982. The ecology of leaf life spans. Annual Review of Ecology and Systematics 13: 229-259.

Chen, J.-W.; Zhang, Q. \& Cao, K.-F. 2009. Inter-species variation of photosynthetic and xylem hydraulic traits in the deciduous and evergreen Euphorbiaceae tree species from a seasonally tropical forest in south-western China. Ecological Research 24: 65-73.

Cordell, S.; Goldstein, G.; Meinzer, F.C. \& Vitousek, P.M. 2001. Regulation of leaf life-span and nutrientuse efficiency of Metrosideros polymorpha trees at two extremes of a long chronosequence in Hawaii. Oecologia 127: 198-206.

Cornelissen, J.H.C.; Lavorel, S.; Garnier, E.; Diaz, S.; Buchmann, N.; Gurvich, D.E.; Reich, P.B.; ter Steege, H.; Morgan, H.D. \& van der Heijden, M.G.A. 2003. A handbook of protocols for standardized and easy measurement of plant functional traits worldwide. Australian journal of Botany 51: 335-380.

Coste, S.; Baraloto, C.; Leroy, C.; Marcon, É.; Renaud, A.; Richardson, A.D.; Roggy, J.C; Schimann, H.; Uddling, J. \& Hérault, B. 2010. Assessing foliar chlorophyll contents with the SPAD-502 chlorophyll meter: a calibration test with thirteen tree species of tropical rainforest in French Guiana. Annals of Forest Science 67: 607.

Damesin, C.; Rambal, S. \& Joffre, R. 1998. Cooccurrence of trees with different leaf habit: A functional approach on Mediterranean oaks. Acta Oecologica 19: 195-204.

Dudley, S.A. 1996. Differing selection on plant physiological traits in response to environmental water availability: a test of adaptive hypotheses. Evolution 50: 92-102.

Eamus, D. 1999. Ecophysiological traits of deciduous and evergreen woody species in the seasonally dry tropics. Trends in Ecology \& Evolution 14: 11-16.

Franco, A.C.; Bustamante, M.; Caldas, L.S.; Goldstein, G.; Meinzer, F.C.; Kozovits, A.R.; Rundel, P. \& Coradin, V.T.R. 2005. Leaf functional traits of Neotropical savanna trees in relation to seasonal water deficit. Trees 19: 326-335. 
Fu, P.L.; Jiang, Y.J.; Wang, A.Y.; Brodribb, T.J.; Zhang, J.L.; Zhu, S.D. \& Cao, K.F. 2012. Stem hydraulic traits and leaf water-stress tolerance are co-ordinated with the leaf phenology of angiosperm trees in an Asian tropical dry karst forest. Annals of Botany 110: 189-199.

Givnish, T.J. 2002. Adaptive significance of evergreen vs. deciduous leaves: solving the triple paradox. Silva Fennica 36: 703-743.

Hammer, O.; Harper, D.A.T. \& Ryan, P.D. 2001. PAST: paleontological statistics software package for education and data analysis. Paleontologia Electronica 4: 1-9.

Heschel, M.S. \& Riginos, C. 2005. Mechanisms of selection for drought stress tolerance and avoidance in Impatiens capensis (Balsaminaceae). American Journal of Botany 92: 37-44.

Ishida, A.; Diloksumpun, S.; Ladpala, P.; Staporn, D.; Panuthai, S.; Gamo, M.; Yazaki, K.; Ishizuka, M. \& Puangchit, L. 2006. Contrasting seasonal leaf habits of canopy trees between tropical dry-deciduous and evergreen forests in Thailand. Tree Physiology 26: 643-656.

Ishida, A.; Nakano, T.; Yazaki, K.; Matsuki, S.; Koike, N.; Lauenstein, D.L.; Shimizu, M. \& Yamashita, N. 2008. Coordination between leaf and stem traits related to leaf carbon gain and hydraulics across 32 drought-tolerant angiosperms. Oecologia 156: 193-202.

Ishida, A.; Harayama, H.; Yazaki, K.; Ladpala, P.; Sasrisang, A.; Kaewpakasit, K.; Panuthai, S.; Staporn, D.; Maeda, T.; Gamo, M., Diloksumpun, S.; Puangchit, L. \& Ishizuka, M. 2010. Seasonal variations of gas exchange and water relations in deciduous and evergreen trees in monsoonal dry forests of Thailand. Tree Physiology 30: 935-945.

Kikuzawa, K. 1991. A Cost-Benefit Analysis of Leaf Habit and Leaf Longevity of Trees and Their Geographical Pattern. The American Naturalist 138: 1250-1263.

Kogami, K.; Hamba, Y. T.; Kibe, T.; Terashima, I.; Masuzawa, T. 2001. $\mathrm{CO}_{2}$ transfer conductance, leaf structure and carbon isotope composition of Polygonum cuspidatum leaves from low and high altitudes. Plant Cell Environment 24: 529-538.

Levitt, J. 1972. Responses of plants to environmental stresses. Academic Press, New York. Pp. 732.

Lima, A.L.A. \& Rodal, M.J.N. 2010. Phenology and wood density of plants growing in the semi-arid region of northeastern Brazil. Journal of Arid Environments 74: 1363-1373.

Lima, A.L.A.; Sampaio, E.V.D.S.B.; De Castro, C.C.; Rodal, M.J.N.; Antonino, A.C.D. \& Melo, A.L. 2012. Do the phenology and functional stem attributes of woody species allow for the identification of functional groups in the semiarid region of Brazil? Trees 26: 1605-1616.
Mantovani, A. 1999. A method to improve leaf succulence quantification. Brazilian Archives of Biology and Technology 42: 9-14.

Mediavilla, S. \& Escudero, A. 2003. Stomatal responses to drought at a Mediterranean site: a comparative study of co-occurring woody species differing in leaf longevity. Tree Physiology 23: 987-996.

Odening, W.R.; Strain, B.R. \& Oechel, W.C. 1974. The effect of decreasing water potential on net $\mathrm{CO} 2$ exchange of intact desert shrubs. Ecology 55: 1086-1095.

Peel, M.C. Finlayson, B.L. \& McMahon, T.A. 2007. Updated world map of the Köppen-Geiger climate classification. Hydrology and Earth System Sciences 11: 1633-1644.

Oliveira, C.C.; Zandavalli, R.B.; Lima, A.L.A. \& Rodal, M.J.N. 2015. Functional groups of woody species in semi-arid regions at low latitudes. Austral Ecology 40: 40-49.

Poorter, L. 2009. Leaf traits show different relationships with shade tolerance in moist versus dry tropical forests. New Phytologist 181: 890-900.

R Development Core Team. 2008. R: A language and environment for statistical computing. R Foundation for Statistical Computing, Vienna.

Reich, P.B.; Walters, M.B. \& Ellsworth, D.S. 1992. Leaf life-span in relation to leaf, plant, and stand characteristics among diverse ecosystems. Ecological monographs 62: 365-392.

Silva, E.C.; Nogueira, R.J.M.C.; Azevedo Neto, A.D.; Brito, J.Z. \& Cabral, E.L. 2004. Aspectos ecofisiológicos de dez espécies em uma área de caatinga no município de Cabaceiras, Paraíba, Brasil. Iheringia Série Botânica 59: 201-205.

Silva, A.M.L.; Lopes, S.F.; Vitorio, L.A.P.; Santiago, R.R.; Mattos, E.A. \& Trovão, D.M.B.M. 2014. Plant functional groups of species in semiarid ecosystems in Brazil: wood basic density and SLA as an ecological indicator. Brazilian Journal of Botany 37: 229-237.

Sobrado, M.A. 1993. Trade-off between water transport efficiency and leaf life-span in a tropical dry forest. Oecologia 96: 19-23.

Trovão, D.M.B.M.; Fernandes, P.D.; Andrade, L.A. \& Neto, J.D. 2007. Variações sazonais de aspectos fisiológicos de espécies da Caatinga. Revista Brasileira de Engenharia Agrícola e Ambiental 11: 307-311.

Williams-Linera, G. 2000. Leaf demography and leaf traits of temperate-deciduous and tropical evergreen-broadleaved trees in a Mexican montane cloud forest. Plant Ecology 149: 233-244.

Wilson, P.J.; Thompson, K.E.N. \& Hodgson, J.G. 1999. Specific leaf area and leaf dry matter content as alternative predictors of plant strategies. New Phytologist 143: 155-162. 
Wright, I.J.; Leishman, M.R.; Read, C. \& Westoby, M. 2006. Gradients of light availability and leaf traits with leaf age and canopy position in 28 Australian shrubs and trees. Functional Plant Biology 33: 407-419.

Wright, I.J.; Reich, P.B.; Westoby, M.; Ackerly, D.D.; Baruch, Z.; Bongers, F.; Cavender-Bares, J.; Chapin, T.; Cornelissen, J.H.C.; Diemer, M.; Flexas, J.; Garnier, E.; Groom, P.K.; Gulias, J.; Hikosaka, K.; Lamont, B.B.; Lee, T.; Lee, W.; Lusk, C.; Midgley, J.; Navas, M.L.; Niinemets,
Ü.; Oleksyn, J.; Osada, N.; Poorter, H.; Poot, P.; Prior, L., Pyankov, V.; Roumet, C.; Thomas, S.; Tjoelker, M.G.; Veneklaas, E.J. \& Villar, R. 2004. The worldwide leaf economics spectrum. Nature 428: 821-827.

Zanne, A.E.; Westoby, M.; Falster, D.S.; Ackerly, D.D.; Loarie, S.R.; Arnold, S.E.J; Coomes, D.A. 2010. Angiosperm wood structure: Global patterns in vessel anatomy and their relation to wood density and potential conductivity. American Journal of Botany 97: 207-215. 